\title{
Pre-pubic phalloplasty by abdominal wall z-plasty for epispadias repair
}

\begin{abstract}
Patients with bladder exstrophy-epispadias complex are known with several penile malformations. A 21-year old patient, with successful exstrophy-epispadias repair as a child, complained of a distinct, dorsal angulation of his penis in erection, making intercourse impossible. A pre-pubic abdominal wall Z-plasty was performed to widen the pre-pubic area with an excellent, functional result.
\end{abstract}

Keywords: exstrophy, phalloplasty, z-plasty
Volume 3 Issue 2 - 2016

\section{Baten E, Guelinckx P, van Renterghem \\ Departement of Urology, Jessa Ziekenhuis, Belgium}

Correspondence: Evert Baten, Jessaziekenhuis Hasselt, Belgium,Tel 0032472643366,Email Evert.baten@gmail.com

Received: December 08, 2015 | Published: February 17, 2016

\section{Introduction}

Bladder exstrophy-epispadias complex can cause many problems during adult life. Our patient presented with a severe dorsal angulation of his penis in erection, making intercourse impossible. We present an innovative technique, in which we use a prepubic abdominal Z-plasty to correct the angulation, which has never been described before.

\section{Case presentation}

A 21-year old patient complained of a distinct, dorsal erectile angulation, making intercourse impossible. He underwent augmentation cystoplasty with appendico-vesicostomy (Mitrofanoff) and epispadias-repair for a bladder exstrophy-epispadias defect as a child. He has normal sensitivity, ejaculations and a good, erectile rigidity in his small $(7 \mathrm{~cm})$ penis.

During surgery an artificial erection was performed with physiologic serum injected in the cavernosal bodies.

With the aid of the plastic surgeon, an infra-umbilical, abdominal wall Z-plasty was performed. The skin and subcutaneous tissue were mobilized to widen the pre-pubic area and to diminish the dorsal angulation. As such an excellent result was achieved and the erectile angulation was reduced, making intercourse possible. The patient returned home the next day and was very satisfied with the functional and aesthetic result (Figure 1-3).

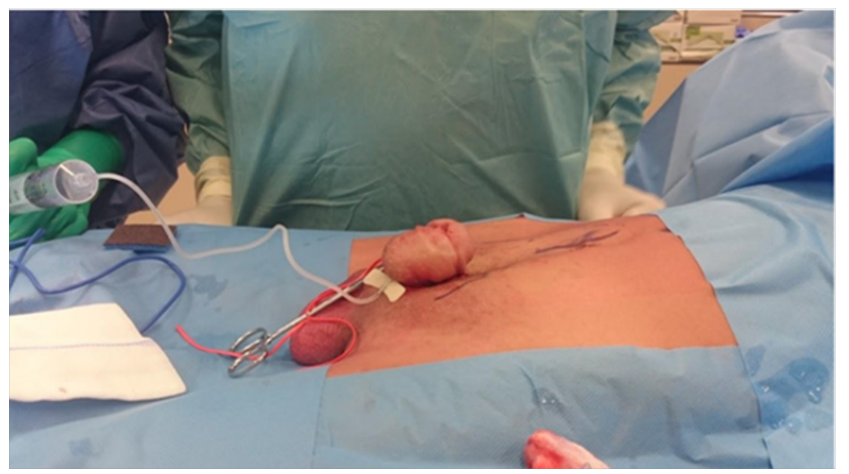

Figure I Artificial erection showing a distinct angulation of the penis.

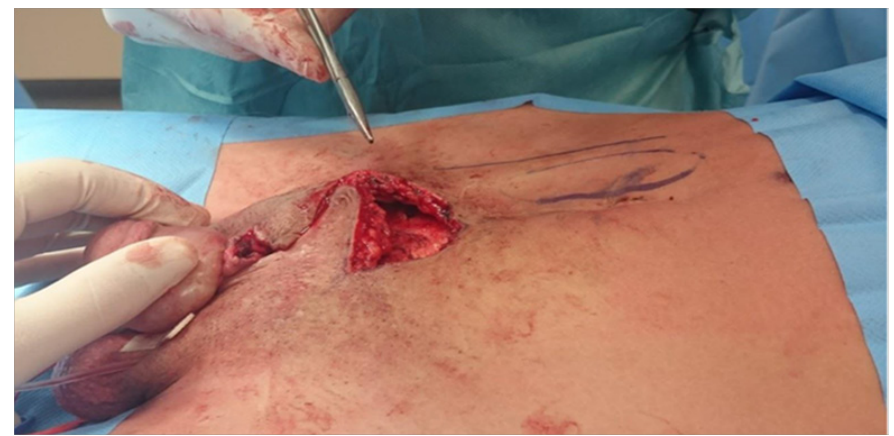

Figure 2 Abdominal wall Z-plasty.

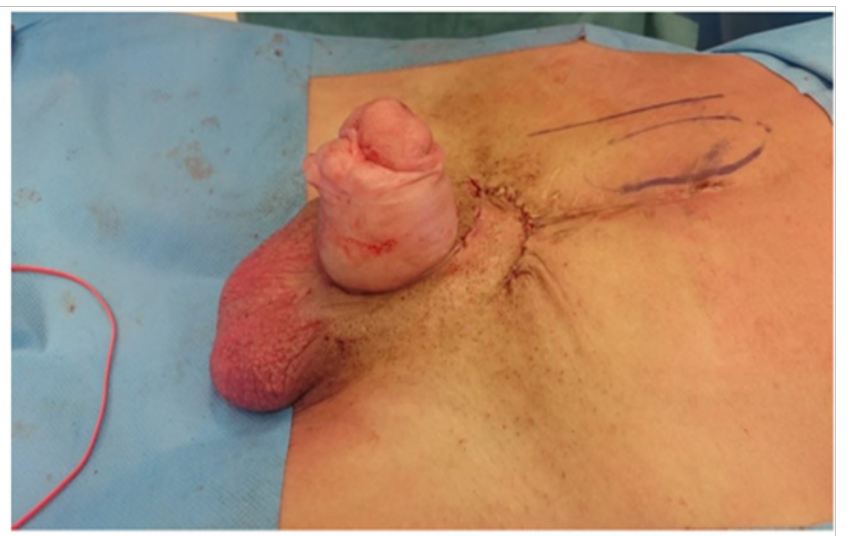

Figure 3 Reduction of the erectile angulation.

\section{Discussion}

Bladder exstrophy is a rare malformation, characterized by an infra-umbilical abdominal wall defect, epispadias, alterations in pubic bone and incomplete closure of the bladder. ${ }^{1}$ The most popular theory explaining the exstrophy-epispadias defect describes an overgrowth of the cloacal membrane that prevents medial migration of the mesenchymal tissues. ${ }^{1,2}$ The incidence is 1 in $30-50,000$ for bladder exstrophy and 1 in 100,000 for epispadias. 
Historically, it was thought that the pubic diastasis was the sole reason for the shorter penis in exstrophy. The Johns Hopkins group however has shown that these patients also have a significant reduction in corporeal tissue mass compared to normal. ${ }^{3,4}$ Our patient had a small but well-functioning penis, concerning sensitivity and erectile rigidity but with a very steep angulation. An extensive search in literature was performed for similar cases but none were found. Our solution compromises an easy to perform solution with an abdominal wall Z-plasty to widen the pre-pubic area and to diminish as such the dorsal angulation.

\section{Acknowledgements}

None.

\section{Conflict of interest}

The author declares no conflict of interest.

\section{References}

1. Pierre K, Borer J, Phelps A, et al. Bladder exstrophy: current management and postoperative imaging. Pediatr Radiol. 2014;44(7):768-786.

2. Muecke EC. The role of the cloacal membrane in exstrophy: the first successful experimental study. J Urol. 1964;92:659-667.

3. Wood D, Woodhouse C. Penile anomalies in adolescence. TSW Urology. 2011;11:614-623.

4. Woodhouse CR, Kellett MJ. Anatomy of the penis and its deformities in exstrophy and epispadias. J Urol. 1984;132(6):1122-1124. 